\title{
Dynamo action in turbulent flows
}

\author{
V. Archontis ${ }^{1}$, S. B. F. Dorch ${ }^{2}$, and Å. Nordlund ${ }^{2}$ \\ 1 Instituto de Astrofisica de Canarias, Via Lactea s/n, 38200 La Laguna, Spain \\ 2 The Niels Bohr Institute for Astronomy, Physics and Geophysics, Juliane Maries Vej 30, 2100 Copenhagen Ø, Denmark
}

Received 4 June 2003 / Accepted 21 August 2003

\begin{abstract}
We present results from numerical simulations of nonlinear MHD dynamo action produced by three-dimensional flows that become turbulent for high values of the fluid Reynolds number. The magnitude of the forcing function driving the flow is allowed to evolve with time in such way as to maintain an approximately constant velocity amplitude (and average kinetic energy) when the flow becomes hydrodynamically unstable. It is found that the saturation level of the dynamo increases with the fluid Reynolds number (at constant magnetic Prandtl number), and that the average growth rate approaches an asymptotic value for high fluid Reynolds number. The generation and destruction of magnetic field is examined during the laminar and turbulent phase of the flow and it is found that in the neighborhood of strong magnetic flux "cigars" Joule dissipation is balanced by the work done against the Lorentz force, while the steady increase of magnetic energy occurs mainly through work done in the weak part of the magnetic field.
\end{abstract}

Key words. magnetic fields - magnetohydrodynamics (MHD) - turbulence - diffusion

\section{Introduction}

The dynamo problem is mainly related to the understanding of the processes of magnetic field generation due to the motions of conducting fluids, the so-called dynamo action (Cowling 1934; Moffatt 1978; Parker 1979). One often divides dynamo theory into two theoretical regimes: first, the kinematic dynamo problem in which magnetic fields and flows are decoupled. The velocity of the flow is prescribed and the evolution of the magnetic field is governed by the induction equation (here in dimensionless form):

$\frac{\partial \boldsymbol{B}}{\partial t}=\nabla \times(\boldsymbol{u} \times \boldsymbol{B})+\frac{1}{R e_{\mathrm{m}}} \nabla^{2} \boldsymbol{B}$,

where $\boldsymbol{B}$ is the magnetic field and $\boldsymbol{u}$ is the prescribed velocity field. The quantity $R e_{\mathrm{m}}=u \ell / \eta$ is the magnetic Reynolds number and it is often huge in astrophysical systems, where $u$ and $\ell$ are characteristic velocity and length scales and $\eta$ is the magnetic diffusivity. A flow acts as a fast dynamo if the growth rate does not tend to zero in the limit of infinite magnetic Reynolds number. The solar dynamo is an astrophysical example of fast dynamo action since it operates on the convective time scale of the fluid which is fast compared to that of the Ohmic diffusion. In the fast kinematic dynamo problem one seeks velocity fields that lead to the exponential amplification of the magnetic field through induction Eq. (1). A powerful mechanism for growth of the magnetic field is the stretching of the

Send offprint requests to: V. Archontis, e-mail: vasilis@ll.iac.es magnetic field lines (Archontis et al. 2003), and is primarily achieved by chaotic flows. The kinematic approach is valid when the magnetic field is weak and there are no further dynamical effects. In the second, fully dynamical dynamo regime, the velocity field is not prescribed and the exponential growth of the magnetic field saturates when the field becomes strong enough to modify the flow sufficiently through the feedback by the Lorentz force. The saturation state of the dynamo then emerges as a self consistent solution to the equations of nonlinear magneto-hydrodynamics. In a fully self-consistent dynamical experiment the forcing would have internal causes, e.g. of convective origin-here we are concerned with the case of an externally prescribed forcing function.

The nonlinear properties of fast dynamos have received considerable attention and a variety of both analytical and numerical studies have provided a valuable insight into the nature of the dynamo action and saturation (Nordlund et al. 1992; Brandenburg et al. 1995; Cattaneo et al. 1996; Zienicke et al. 1998; Brummell et al. 1998). However, a difficulty that arises in numerical simulations is that often, fast dynamo flows become hydrodynamically unstable at fluid Reynolds number ( $R e=u \ell / v, v$ being the viscosity) greater than a critical value (Podvigina \& Pouquet 1994) and spatio-temporal turbulence appears to be nascent for higher values of Re. As a result the desired flow velocity is modified even in the kinematic regime and the problem contains the complexity of that of a turbulent dynamo. Thus, the choice of the forcing function that drives the flow is crucial. We use a forcing function with an amplitude 
that is allowed to evolve with time, keeping the average kinetic energy approximately constant through both laminar and turbulent phases. The growth rates and the saturation level of such turbulent dynamos are examined with respect to the magnetic and fluid Reynolds numbers. Also of great interest is the understanding of the processes at work in the kinematic (both laminar and turbulent phase of the flow) and in the saturated regime. We present an analysis of the Lorentz work and Joule dissipation, which shows that they are in close balance and that dynamo-work occurs mainly in the weak part of the magnetic field where dissipation is insignificant.

The paper is organized as follows. Section 2 contains the equations, the numerical method and a description of the driving of the flow that has been used in the simulation. Results for turbulent dynamo action are presented in Sect. 3. The structures of the velocity field and the magnetic field in physical space are discussed in Sect. 4. Whether or not the growth rates and the saturation level of the dynamo depend on the magnetic or fluid Reynolds number is examined in Sects. 5 and 6. A qualitative understanding of the nature of the dynamo is discussed in Sect. 7, using results from a kinematic dynamo experiment. Section 8 contains the overall conclusions of the present numerical simulations.

\section{The simulation}

\subsection{The equations}

The compressible MHD equations are solved numerically in a periodic computational domain, with periodicity of $2 \pi$ in all three directions. Apart from the magnetic induction equation Eq. (1), the additional equations solved are the following:

$$
\begin{aligned}
& \frac{\partial \rho}{\partial t}=-\nabla \cdot \rho \boldsymbol{u}, \\
& \frac{\partial(\rho \boldsymbol{u})}{\partial t}=-\nabla P+\boldsymbol{j} \times \boldsymbol{B}+\boldsymbol{f}-\nabla \cdot(\rho \boldsymbol{u} \boldsymbol{u}), \\
& \frac{\partial e}{\partial t}=-\nabla \cdot(e \boldsymbol{u})-P \nabla \cdot \boldsymbol{u}+Q_{\mathrm{v}}+Q_{\mathrm{J}}+Q_{\mathrm{cool}}
\end{aligned}
$$

where $\rho$ is the fluid density, $P$ the pressure, $\boldsymbol{j}$ is the electric current density, $e$ is the internal energy and $f$ is an external forcing term. $Q_{\mathrm{v}}$ and $Q_{\mathrm{J}}$ are the viscous and Joule dissipation respectively. In the experiments, a Newtonian cooling term, $Q_{\text {cool }}=\left(T-T_{0}\right) / \tau_{\text {cool }}$ is used, where $T$ is the temperature and $\tau_{\text {cool }}=5$. The cooling term only works as a sink of the thermal energy, in order to balance the heat generation by dissipative terms in the energy equation and the actual magnitude of the cooling time is of little importance with no effect on the results of our simulation.

The above equations are solved numerically on a staggered mesh. The time stepping is performed by a third order predictor-corrector method (Hyman 1979). The derivatives and interpolations are of 6th and 5th order respectively and the numerical scheme conserves $\nabla \cdot \boldsymbol{B}=0$ exactly. Numerical solutions are obtained on a grid of a maximum of $180^{3}$ points, using a modified version of the code by Galsgaard, Nordlund and others (Galsgaard \& Nordlund 1997; Nordlund et al. 1992). The initial magnetic field is chosen to be a weak random perturbation with an amplitude of $10^{-5}$ in non-dimensional units.

\subsection{Flow considerations}

There is a considerable body of work in the literature that deals with fast dynamo action in simple, steady and threedimensional flows. In our simulation the velocity is chosen to have vigorous (fast) dynamo properties in the kinematic regime, where the Lorentz force is negligible. One such class of flows, which are well known candidates for fast dynamo action is the $\mathrm{ABC}$ flows. The form of the velocity is given by

$$
\begin{aligned}
\boldsymbol{u}_{\mathrm{ABC}}= & A(0, \sin k x, \cos k x)+B(\cos k y, 0, \sin k y) \\
& +C(\sin k z, \cos k z, 0)
\end{aligned}
$$

This periodic flow is the sum of three steady Beltrami waves, parameterized by $A, B$ and $C$ and has the property $\nabla \times \boldsymbol{u} \propto \boldsymbol{u}$ (velocity is parallel to vorticity). If one or more of the constant coefficients $(A, B$ and $C$ ) is zero, the flow is integrable and is not a fast dynamo. If all three are non-zero the flow is non-integrable (Dombre et al. 1986) and contains a mixture of chaotic regions and regular islands. The special case $A=B=C=1$ was introduced by Childress (1970) as a model for kinematic dynamo action. Arnold et al. (1983) noted that a steady three-dimensional flow with infinite conductivity and chaotic streamlines favors the growth of magnetic fields. Their results were followed by the work of Galloway et al. (1984) and Moffatt et al. (1985) who studied dynamo action in ABC flows with finite conductivity. A detailed description of the amplification process responsible for the kinematic dynamo action, when the wavenumber of the flow $k$ is equal to one or higher, has been provided by Dorch (2000) and Archontis et al. (2003). The hydrodynamic stability of the ABC flow has been studied by Podvigina \& Pouquet (1994) who found that for values of fluid Reynolds number above a critical one $\left(R e_{\mathrm{c}}=13\right)$ the $\mathrm{ABC}$ flow destabilizes, first to time dependent but still smooth states, and then to a turbulent state. In our simulation, the definition of the Reynolds numbers is the same as in Galanti et al. (1992).

In the following experiments the initial flow is taken to be an ABC flow with $A=0.9, B=1, C=1.1$ and wavenumber equal to unity. The reason for choosing values of $A, B$, and $C$ slightly different from unity, is that the $A=B=C$ case is too special, because of its exact symmetry, and thus turbulence takes longer to develop. The purpose of our work is to study dynamo action in the turbulent regime of driven $\mathrm{ABC}$ flows since astrophysical dynamos typically occur in turbulent environments with very high fluid and magnetic Reynolds numbers. However, the behavior of turbulent dynamos in the high $R e$, $R e_{\mathrm{m}}$ limit may be expected to be to some extent generic. An increase in the Reynolds numbers involves smaller and smaller length scales where dissipation actually occurs. However, the rate of dissipation is determined by the large scale developments of the flow and is independent of the magnitude of the viscosity once the Reynolds numbers are large. Thus, there is good hope that also dynamo properties are asymptotically independent of the Reynolds numbers. That hope is indeed borne out by the present numerical experiments, as is discussed in more details below. 


\section{Results}

As a starting point, the forcing function which is imposed is such that the ABC flow Eq. (6) appears. The forcing necessary to accomplish this must have the following form:

$f=-\frac{1}{R_{\mathrm{e}}} \nabla^{2} \boldsymbol{u}_{\mathrm{ABC}}$

However, in order to ensure that the kinetic energy is maintained at a value close to the initial value, the amplitude of the forcing is controlled by a differential equation, which compensates for the increased dissipation in the turbulent phase by increasing the forcing when the kinetic energy tends to drop below the nominal value. The amplitude factor in front of the driving force is given by

$K=\frac{E_{0}}{E_{\text {kin }}} \mathrm{e}^{L}$,

where $L$ is determined from

$\frac{\mathrm{d} L}{\mathrm{~d} t}=\frac{1}{\tau_{\mathrm{t} . \mathrm{o} .}} \ln \frac{E_{0}}{E_{\text {kin }}}$,

with $L(t=0)=0$. The differential equation evolves the amplitude factor $\mathrm{e}^{L}$ on the turn over time scale, $\tau_{\text {t.o. }}$, if the kinetic energy tends to deviate from the nominal value $E_{0}$. The immediate factor $E_{0} / E_{\text {kin }}$ helps to reduce the time delay that results from the integral nature of the exponential factor, and the delayed response of the kinetic energy on changes in the driving. The kinetic energy control is an essential feature, in that otherwise the amplitude of the velocity in the turbulent phase would become arbitrarily small, at increasingly large fluid Reynolds number, since the forcing in the laminar phase needs to scale with $1 / R e$. It is indeed necessary to maintain the kinetic energy in order to maintain the same turn over time and actual fluid Reynolds number, since the formal fluid Reynolds number is defined in terms of the rms velocity of the laminar ABC flow.

Figure 1 shows the evolution of the kinetic energy in time for the first 100 time units. Two phases may be identified. The laminar phase which extends up to $t=30$ and the turbulent regime for $t>30$. Note that the kinetic energy drops by up to about $40 \%$ when the flow enters the turbulent phase. After a short adjustment time, the forcing control returns the kinetic energy to the nominal level, where it is then maintained, except for minor fluctuations.

The magnetic energy for the same time period during the laminar phase increases exponentially and the growth rate is about 0.15 . When the flow enters the turbulent phase $(t>30)$ the magnetic energy drops slightly and later $(t=40)$ continues to grow exponentially with a similar growth rate as in the laminar phase (Fig. 2).

An analysis of the external work in the above two distinct phases shows that the average work in the turbulent regime is much larger than in the laminar case (Fig. 3). On the first hand, the asymptotic level of the average work $(\approx 0.25)$ is independent of $R e$ and incidentally corresponds closely to the level that would be obtained for the critical value $\left(R e_{\mathrm{c}}\right)$. On the other hand, the average work in the laminar regime is proportional to $1 / R e$, and can become arbitrarily small for high enough Reynolds numbers.

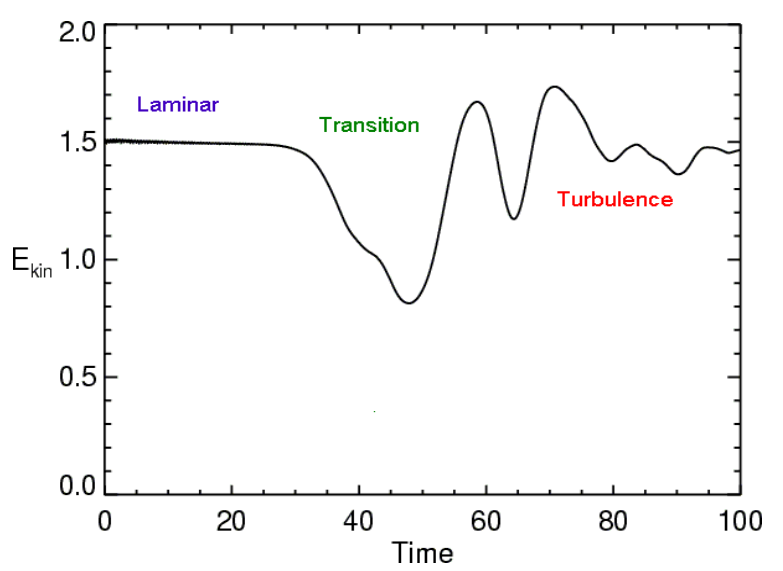

Fig. 1. Temporal evolution of the kinetic energy showing the transition from a laminar to a turbulent state.

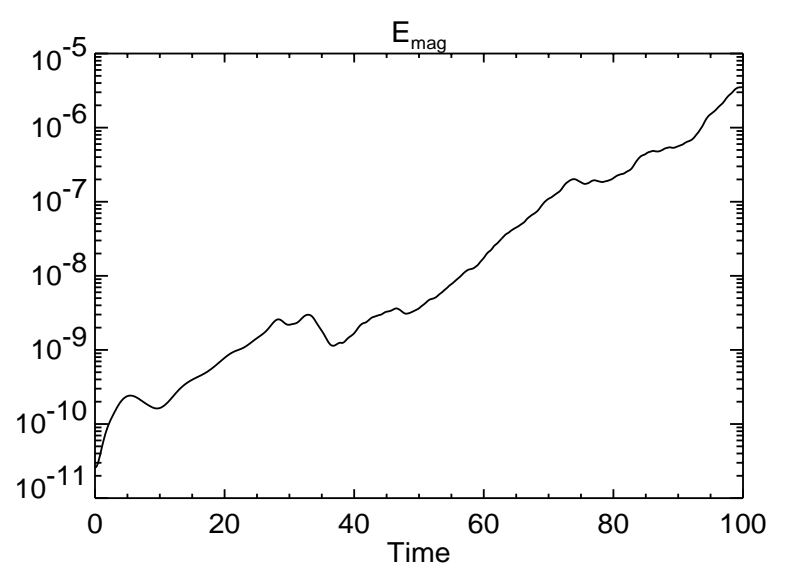

Fig. 2. Temporal evolution of the magnetic energy during the kinematic regime of the dynamo.

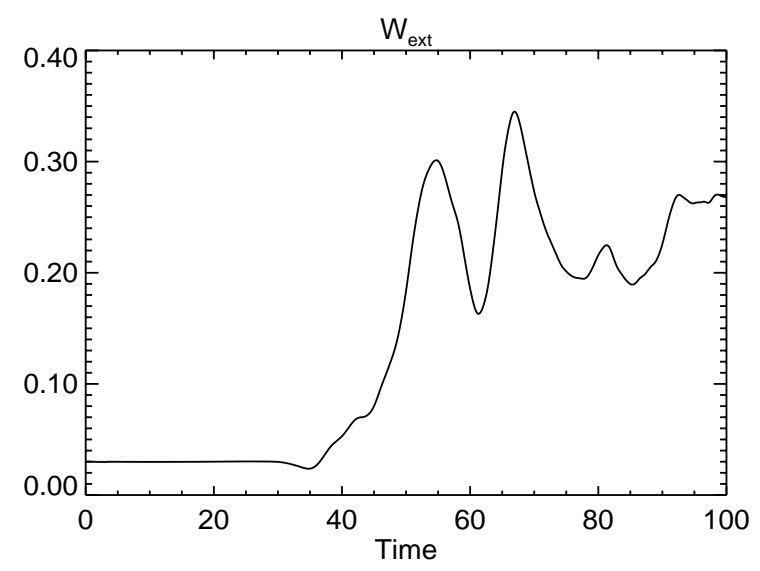

Fig. 3. The evolution of the external average work in time.

This illustrates the point made above, about the forcing control; without it, the amplitude of the velocity in the turbulent phase would drop very much and the kinetic energy could not be maintained in order to have constant fluid Reynolds number during the simulation.

The enhancement of the driving force above the laminar value is shown in Fig. 4. The factor in Eq. (7) is by definition = 1 during the laminar phase of the flow but increases during the 


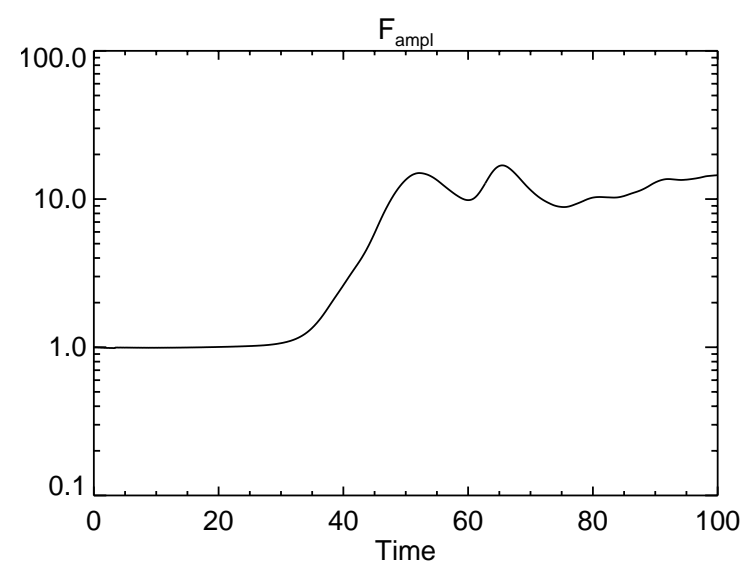

Fig. 4. The increase of the amplitude of the driving force going from the laminar phase to the turbulent phase.

turbulent phase to maintain the kinetic energy. The asymptotic level of the factor is about 10 for $R e=100$.

Figure 5 shows the viscous dissipation as a function of time for the laminar and turbulent phase. During the laminar phase the dissipation is constant and is balanced by the external work. The viscous dissipation increases when the flow becomes turbulent. It reaches an asymptotic level when the turbulent velocity is maintained at the same level as that of the laminar flow.

Figure 6 compares the Joule dissipation to the work done against the Lorentz force and also shows the temporal evolution of the magnetic energy for the kinematic regime of the dynamo.

The work done against the Lorentz force is leading the Joule dissipation and is ultimately responsible for the increase in the magnetic energy. There is a close balance between them with a small positive difference during the increase of the magnetic energy and small negative difference when the magnetic energy decreases, see Figs. 6a,b. The fluctuations of the Lorentz work lead similar fluctuations of the Joule dissipation when the magnetic energy increases or decreases. Their difference in the saturated state of the dynamo fluctuates around zero, as expected. The work done by the fluid on the magnetic field is, on average, converted into Joule heat and none is left to increase the magnetic energy.

The accuracy in the numerical method is demonstrated in Fig. 6c, where the $\mathrm{d} E_{\mathrm{mag}} / \mathrm{d} t$ is overplotted (symbols) on top of the difference $W_{\mathrm{L}}-Q_{\mathrm{J}}$. These are averages over the periodic box, for which the flux term in the magnetic energy equation vanish. The magnetic energy equation is

$\frac{\partial E_{\mathrm{mag}}}{\partial t}=-\nabla \cdot \boldsymbol{F}_{\mathrm{poynt}}-\boldsymbol{u} \cdot \boldsymbol{f}_{\mathrm{L}}-Q_{\mathrm{J}}$

The source of magnetic energy is the work done against the Lorentz force and the sink is the Joule dissipation. Magnetic energy is transported by the Poynting flux, $\boldsymbol{F}_{\text {poynt }}=\boldsymbol{E} \times \boldsymbol{B}$. According to Eq. (9), $\mathrm{d} E_{\mathrm{mag}} / \mathrm{d} t$ should be identically equal to $W_{\mathrm{L}}-Q_{\mathrm{J}}$, and the two are indeed very nearly the same.

Note that the ratio of the Lorentz work to the Joule dissipation in Fig. 6d remains close to unity both during intervals of time when the magnetic energy is growing and during intervals of time when the magnetic energy is decaying.

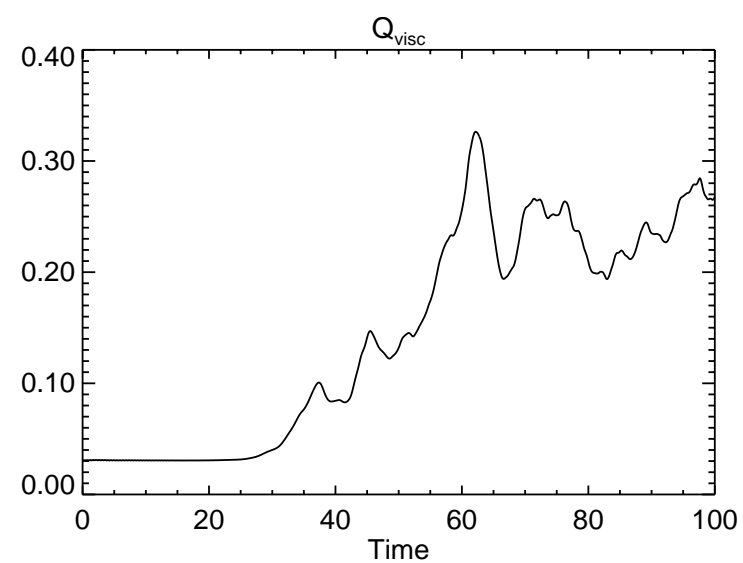

Fig. 5. The evolution of the viscous dissipation up to $t=100$.

\section{Magnetic and velocity patterns}

During the laminar phase the flow has points with twodimensional stable manifolds (so called $\alpha$-type stagnation points), where the magnetic field is concentrated along magnetic "flux cigars". There are also points with two-dimensional unstable manifolds, similar to the $\beta$-type stagnation points of the $A=B=C=1$ flow, where magnetic flux sheets are formed (see Dorch 2000).

Figure 7 (left panel) shows that flux cigars form an inclined triangle around a $\beta$-type stagnation point (located at the center of the box). Another one, which is located at the upper left corner, is pointing towards the plane of the $\beta$-type point while the flow is still laminar. The right panel of the same figure shows low velocity and high magnetic field isosurfaces at $t=30$. The velocity field comes into a turbulent phase and the regions of low velocity are disturbed, but the triangle formed by the flux cigars is still visible. The low velocity isosurfaces no longer contain points of exact stagnation, but still correspond to regions of vigorous stretching (see Archontis et al. 2003), which is why we choose to visualize them.

Figure 8 is a visualization of the same velocity and magnetic field strength for $t=80$. The velocity field has a more turbulent configuration and the regions with velocities less that $4 \%$ of the peak have disappeared. The magnetic flux cigars also disappear and the magnetic energy is confined into curved tubes and weaker sheets.

\section{Saturation of the dynamo}

Two experiments were performed to show the dependence of the saturation level of the turbulent dynamo on the magnetic and fluid Reynolds number. Cases with $R e_{\mathrm{m}}=R e=200$ and $R e_{\mathrm{m}}=R e=400$ are studied and the temporal evolution of the magnetic energy is followed in the kinematic and saturated regime.

Figure 9 shows the growth of the magnetic energy in the beginning of the kinematic regime for $R e_{\mathrm{m}}=R e=200$ and $R e_{\mathrm{m}}=R e=400$. The numerical resolution used were $80^{3}$ and $160^{3}$, respectively. After an initial transient phase the growth rate for the second experiment is very similar to the growth rate of the first one. The difference in the magnetic 

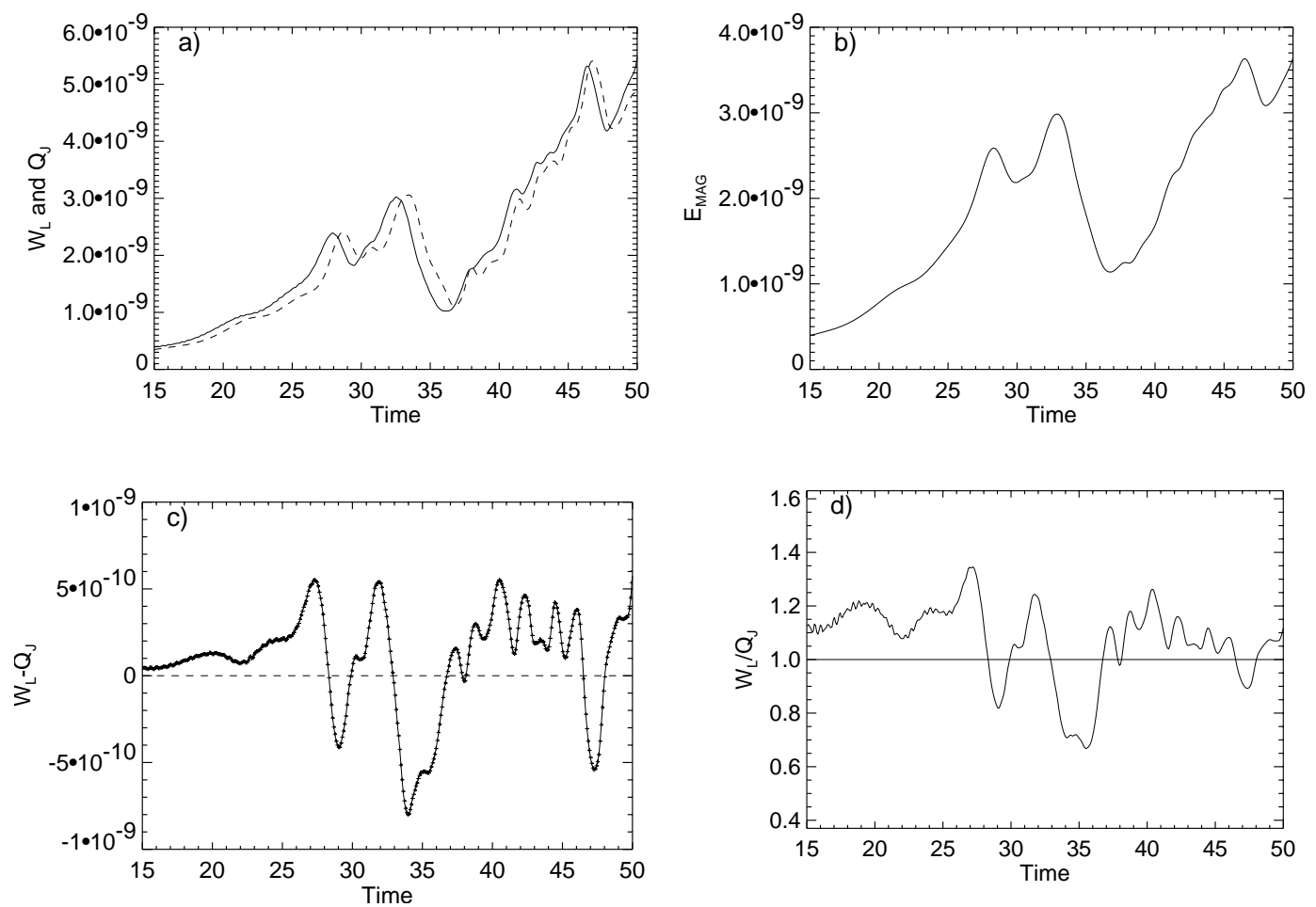

Fig. 6. Four panels showing different aspects of the time evolution of the Lorentz work and Joule dissipation during the linear regime of the dynamo and comparison with the magnetic energy: a) the Lorentz work $W_{\mathrm{L}}$ (full curve) and the Joule dissipation $Q_{\mathrm{J}}$ (dashed curve). b) Total magnetic energy $E_{\mathrm{mag}}$. c) The difference between the Lorentz work and the Joule dissipation. d) Ratio between work and heating.
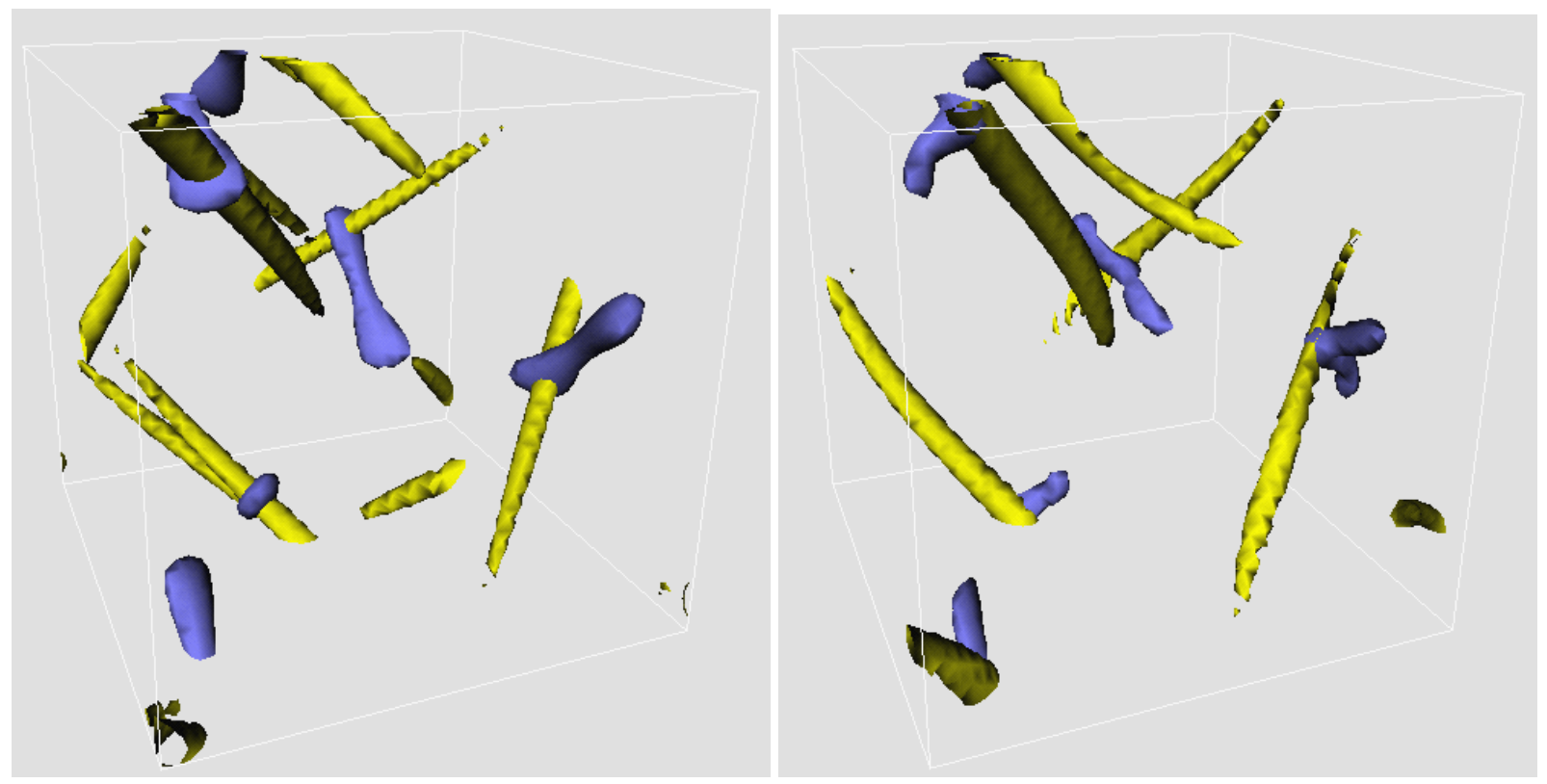

Fig. 7. Isosurfaces of magnetic field strength (light) and low velocity (dark) at $t=20$ (left panel) and $t=30$ (right panel). The isosurface level of the magnetic field strength is $75 \%$ of the peak value in the snapshot. The velocity isosurfaces correspond to $4 \%$ of the peak velocity in each snapshot.

energy amounts to a factor of 2.7. Thus if the magnetic energy for the $160^{3}$ experiment is divided with 2.7 , it falls right on top of the $80^{3}$ experiment with $R e_{\mathrm{m}}=100$ (see Fig. 9).

Keeping the same values of magnetic and fluid Reynolds number, the temporal evolution of the magnetic energy is examined in the saturated regime. The magnetic energy eventually stops growing exponentially and saturates. A result of considerable significance is that for higher values of the fluid Reynolds number, but constant magnetic Prandtl number $\left(P r_{\mathrm{m}}=v / \eta\right)$, the saturation level of the turbulent dynamo increases, rather than decreases (Vainshtein \& Cattaneo 1992).

Figure 10 shows the temporal evolution of the magnetic energy over a small epoch of the non-linear regime, illustrating the effect of increased numerical resolution. There is an initial 


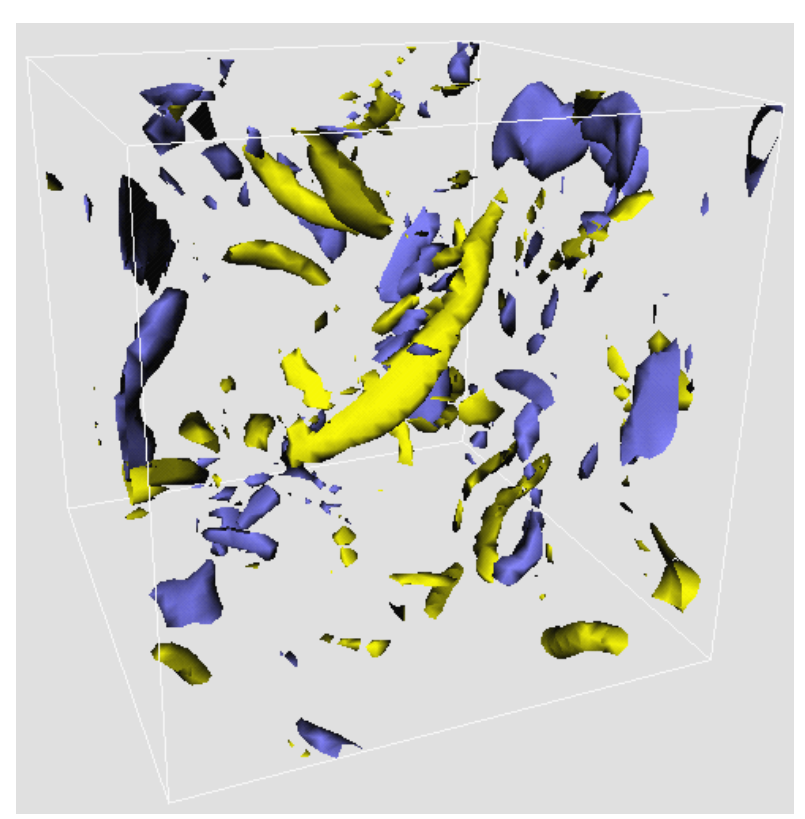

Fig. 8. Isosurfaces of magnetic field strength (light) and low velocity (dark) at $t=80$.

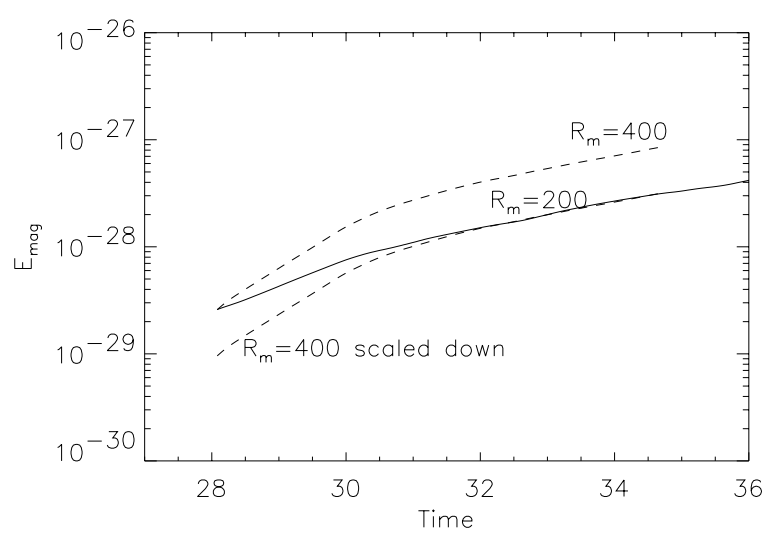

Fig. 9. The growth of the magnetic energy in the kinematic regime when $R e_{\mathrm{m}}=R e=200$ (straight line) at $80^{3}$ resolution and $R e_{\mathrm{m}}=$ $R e=400$ (dashed line) at $160^{3}$ resolution. The latter divided by 2.7 is shown by the lower (dashed) line.

transient, as the larger resolution allows field concentrations to collapse further. Note that there are then sections of time where the growth rates and the decay rates are quite similar in the $R e_{\mathrm{m}}=400$ and $R e_{\mathrm{m}}=200$ cases. The sections after the largest maximum in the two cases, and the rise towards the next maximum are two examples. This might be fortuitous, but more likely it corresponds to parallel evolution of similar magnetic structures.

The overall level is slightly higher, and the evolution is slightly delayed in the high $R e$ case, because flux structures that were barely resolved at $80^{3}$ are allowed to collapse in the $160^{3}$ case. This is indeed also what happens in the kinematic case, but here the size of most structures is controlled by the Lorentz force, and not by diffusion, so the effect is only marginal. For the same reason, the effect may be expected to vanish altogether as the magnetic Reynolds number goes to infinity. In addition, the size of the magnetic structures in the

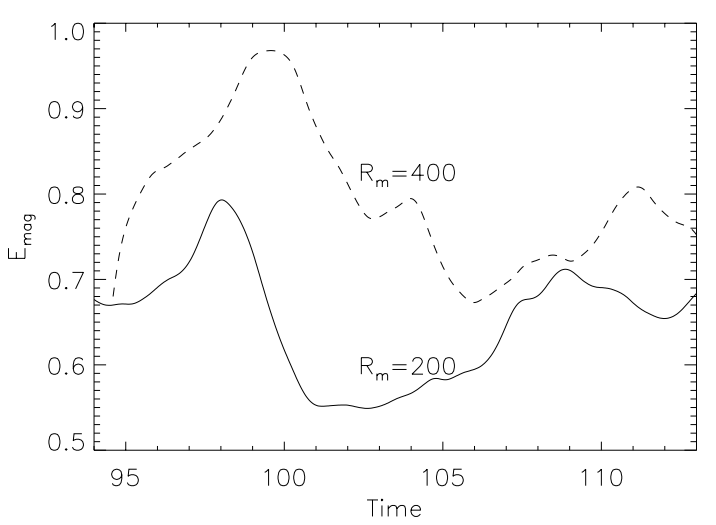

Fig. 10. Temporal evolution of the magnetic energy in the saturated regime for $R e_{\mathrm{m}}=200, R e=200$ (solid line) and $R e_{\mathrm{m}}=400, R e=400$ (dashed line).

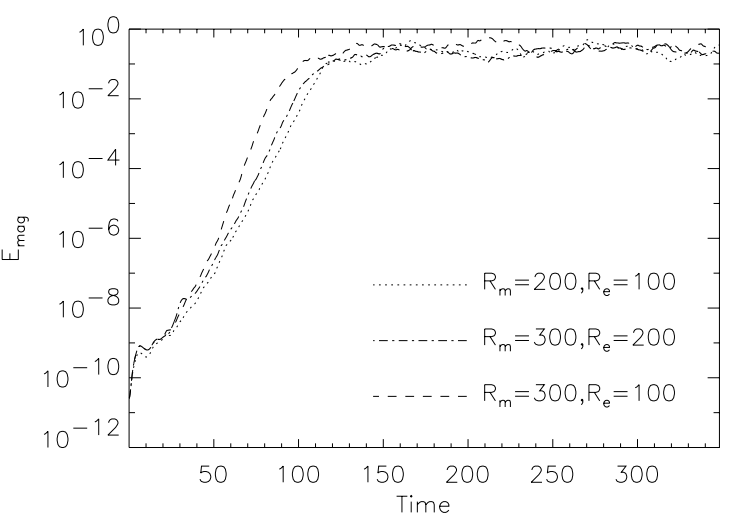

Fig. 11. Temporal evolution of the magnetic energy for $P r_{\mathrm{m}} \geq 1$. Numerical resolution is $96^{3}$ for $R e_{\mathrm{m}}=300$ and $80^{3}$ for $R e_{\mathrm{m}}=200$.

kinematic regime scales as $R e_{\mathrm{m}}^{-1 / 2}$. In the saturation regime the size is again determined by the balance between the advection and dissipation of the magnetic energy and presumably changes following the same scaling with no crucial dependence on the value of $R e$.

After some time, the saturation level for $R e_{\mathrm{m}}=400$, indeed fluctuates around a level that is only marginally larger than for $R e_{\mathrm{m}}=200$. This is small compared to the factor 2.7 for the kinematic regime of the dynamo.

One also notices that there are more fluctuations in the higher resolution case (e.g. the extra bump on the way down after the largest maximum), as a result of the small scales resolved when going to higher $R e_{\mathrm{m}}$. That trend is expected to continue with increasing magnetic Reynolds number.

\section{Magnetic Prandtl number and growth rate}

In Sect. 5 we examined the growth rate and the saturation level of a fast dynamo when the magnetic Prandtl number is equal to one. Equally important is the study of the degree of their dependence when $P r_{\mathrm{m}}$ is varied. Thus, additional experiments were performed where the forcing again is such that it maintains the kinetic energy at a value close to the initial one, apart from small oscillations.

Not surprisingly, the growth rates during the laminar phase $(t<30)$ of the flow are found to be the same (Figs. 11, 12). 


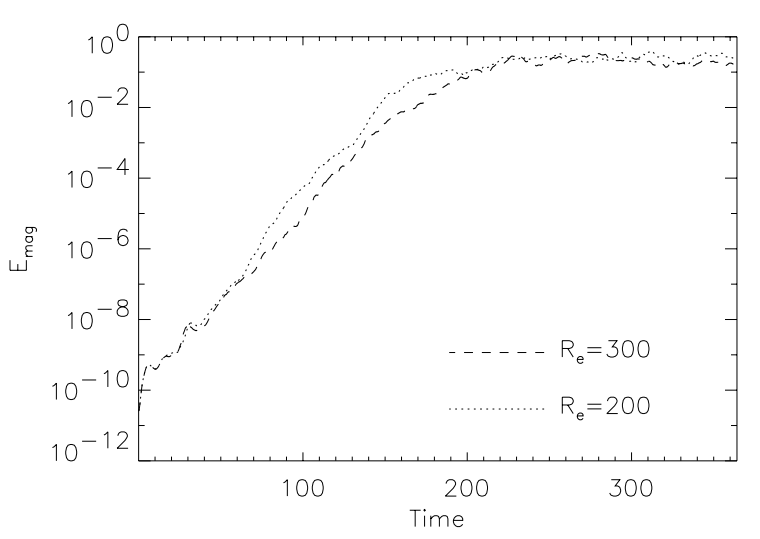

Fig. 12. Temporal evolution of the magnetic energy for $R e_{\mathrm{m}}=200$ and $P r_{\mathrm{m}} \leq 1$.

By construction, the velocity is exactly the same in the laminar phase, independent of $R e$, and the effect of increasing $R e_{\mathrm{m}}$ is simply, as in the comparison of the $R e_{\mathrm{m}}=200$ and $R e_{\mathrm{m}}=400$ experiments, to give a different transient at the beginning of the simulation.

Less trivially, the average growth rates in the turbulent regime also turn out to be similar with no crucial dependence on the magnetic Prandtl number for high $R e$. This is illustrated in Fig. 12, where the difference in the magnetic energy during the turbulent phase $(t>30)$ is very small between the two experiments with $R e=200$ and $R e=300$. This may also be seen as a consequence of the generic properties of high Reynolds number turbulence. More specifically, by one of Kolmogorov's main assumptions, energy dissipation (and hence the forcing) is independent of the fluid viscosity once the Reynolds number is high enough. Thus, and by analogy with the previous experiments, we expect that the only important change in the magnetic field is the development of more fine structure with increasing magnetic Reynolds number. The growth rate is determined by the large scale properties of the flow, and in particular by the turn over time, which by construction does not change between these experiments.

Finally, the above experiments reinforce the conclusion from Sect. 5, that the saturation level of the dynamo does not depend crucially on the value of $P r_{\mathrm{m}}$, but only increases slightly with $R e_{\mathrm{m}}$ as it is shown in Fig. 11 .

\section{Nature of the dynamo}

The results of the above non-linear experiments are similar to the results of previous studies of turbulent hydromagnetic convection (Nordlund et al. 1992) and both results indicate that during the dynamo action there is a close balance between the work done against the Lorentz force and Joule dissipation with a realatively small positive (negative) difference when the magnetic energy increases (decreases). In other words, work and dissipation are in near balance, with a relatively small difference responsible for the average growth of the magnetic energy. One may rightly ask why this is so; is the small positive difference then just fortuitous, and might the difference not just as well be negative for a slightly different flow?
Table 1. Summary of average values from the kinematic experiment with $R e_{\mathrm{m}}=300$.

\begin{tabular}{lc}
\hline \hline Average Lorentz work & 271.1 \\
\hline Average Joule dissipation & 326.3 \\
\hline Average magnetic energy & 332.3 \\
\hline Energy growth rate & 0.166 \\
\hline Fractional dissipation level & 0.0012 \\
\hline Fractional volume & 0.89 \\
\hline Net work in weak dissipation regime & 55.1 \\
\hline Net work in high dissipation regime & 0.09 \\
\hline
\end{tabular}

To address this question we make use of a kinematic dynamo experiment, where the analysis is simpler than in a turbulent case. Turbulence makes the analysis of the Lorentz work difficult - there is much time dependent noise, which corresponds to waves averaging out over time. The work is much less noisy when the velocity is prescribed and/or has not yet entered the turbulent phase, and we therefore analyze the results of an experiment that deals with kinematic dynamo action. The velocity field is chosen to be the $A=B=C=1$ flow with $k=1$ and the magnetic Reynolds number is $R e_{\mathrm{m}}=300$.

The convergence and the diffusion rates at the points of strong flux concentration are of the order of $\sqrt{2}$, which is much larger than the dynamo growth rate (which is about 20 times smaller, e.g. see Childress \& Gilbert 1995), so the advection and diffusion are in close balance in the neighborhood of the "flux cigars". During the exponential growth of the magnetic energy the size of the flux cigars does not change dramatically, but the magnetic energy increases gradually.

A detailed analysis for the latter case confirms that this is indeed the case, and that the bulk part of the Joule dissipation and the work done against the Lorentz force are in detailed balance. This is understandable as a balance between kinetic energy being converted into magnetic energy, that is then immediately converted into heat through Joule dissipation.

Significant net positive work (defined by the difference $W_{\mathrm{L}}-Q_{\mathrm{J}}$ ) occurs instead in regions where the magnetic field is weak, and the dissipation is very weak as well. In these regions, which cover $90 \%$ of the volume in this example, there is almost "pure work"; the Joule dissipation is much smaller than the work, and the net work is almost identical to the net work in the whole box.

In other words: The dissipation is strong only in the neighborhood of the $\alpha$-type stagnation points, but there it is balanced almost exactly by advection/convergence.

Table (1) shows the estimated values of the average work and dissipation over the $89 \%$ of the computational volume. On the one hand, the dissipation is very weak (less than $0.12 \%$ of the maximum dissipation level) and on the other hand, almost all the net work occurs in those $89 \%$ of the volume. Moreover, the largest amount of dissipation and work occur in the remaining $11 \%$ of the volume and they are in almost perfect detailed balance.

The above results lead to the conclusion that dynamo action occurs primarily in regions where the field is weak. 
The net work is much higher than the dissipation and as a result the field is amplified by dynamo action. Most of the net work occurs when the weak field is bent and stretched. The magnetic energy is then distributed over the volume through the Poynting flux and the field lines are dragged out and pile up against the local flux cigars where balance occurs between stretching and diffusion. The weak field is almost perfectly advected with the fluid and this advection of the weak field would be possible even in the saturated regime where the flow still has a good grip on the weakest field, although the strongest field is now exerting a strong resistance against motion/stretching, through the Lorentz force.

The above picture of the dynamo process is most likely related to the observation that the growth rates found for the kinematic ABC dynamos are always consistent with the large scale turn over times, even though the ratio of the magnetic energy to the rate of Joule dissipation $\left(E_{\mathrm{mag}} / Q_{\mathrm{J}}\right)$, which is the time over which the magnetic energy is destroyed and replenished, is much shorter.

In conclusion, the work done on the weak part of the field is responsible for the increase in the magnetic energy by bending and stretching the magnetic field lines. The exponential amplification dominates the Joule dissipation which is weak through almost all of the volume. The detailed balance between the Lorentz work and the dissipation occurs only around the points where strong magnetic field structures are formed. Thus, a dynamo appears to be a process where amplification of the weak field plays the dominant rôle in the growth of the magnetic energy.

\section{Conclusions}

In this paper turbulent dynamo action has been studied and some new aspects of kinematic dynamo action have been illustrated. It was found to be important to keep the kinetic energy level of the same order through the simulation, by automatically adjusting the amplitude of the driving. Using relatively high values of $R e_{\mathrm{m}}$ and $R e$ we find that the growth rate of the magnetic energy amplification apparently depends on the stretching ability of the flow rather than on the values of the above parameters. This is obvious even in the turbulent phase, for different values of the magnetic Prandtl number, where the dissipation is increased. The level at which the magnetic energy saturates increases slightly with $R e_{\mathrm{m}}$ but the transient in the saturated regime is expected to become insignificant at sufficiently high $R e_{\mathrm{m}}$.

An important result is the existence of a near balance between the Lorentz work and the Joule dissipation, which is apparent in both the laminar and the turbulent regime of the forced $\mathrm{ABC}$ flow. Remarkably, the balance originates primarily from small regions where strong magnetic flux structures are concentrated. The nature of the dynamo is such that the net growth of magnetic energy comes about through stretching and folding of the weak magnetic field in the rest of the volume, where dissipation is very weak.

The aim of this paper was not to study dynamo action on a global scale (including aspects such as differential rotation, stratification and convection) but rather to study the different stages of dynamos produced by a family of hydrodynamically unstable flows. However, the physical processes and the dynamo mechanisms studied here are likely to be of similar importance in more realistic stellar dynamo settings.

Acknowledgements. VA thanks the EU-TMR for support through a Marie Curie Fellowship. SBFD was supported by the Danish Natural Science Research Council. The work of $\AA \mathrm{N}$ was supported in part by the Danish National Research Foundation, through its establishment of the Theoretical Astrophysics Center. Access to computational resources granted by the Danish Center for Scientific Computing is gratefully acknowledged.

\section{References}

Archontis, V., Dorch, S. B. F., \& Nordlund, Å. 2003, A\&A, 397, 393

Arnold, V., \& Korkina, E. 1983, Vest. Mosk. Un. Ta. Ser. 1, Matem. Mekh., 3, 43

Beltrami, E. 1889, Opera Matem., 4, 304

Brandenburg, A., Klapper, I., \& Kurths, J. 1995, Phys. Rev. Lett., 52, 4602

Brummell, N. H., Cattaneo, F., \& Tobias, S. M. 1998, Phys. Lett. A, 249,437

Cattaneo, F., Hughes, D. W., \& Kim, E. 1996, Phys. Rev. Lett., 76, 2057

Childress, S. 1970, J. Math. Phys., 11, 3063

Childress, S., \& Gilbert, A. D. 1995, Stretch, Twist, Fold: The Fast Dynamo (Berlin: Springer-Verlag), 52

Cowling, T. J. 1934, MNRAS, 140, 39

Dombre, T., Frisch, U., \& Green, U. 1986, JFM, 167, 353

Dorch, S .B. F. 2000, Phys. Scr., 61, 717

Galanti, B., Pouquet, A., \& Sulem, P. L. 1992, Geophy. \& Astroph. Fluid Dyn., 66, 183-208

Galloway, D., \& Frisch, U. 1984, Geophys. Astroph. Fluid Dyn., 29, 13

Galsgaard, K., \& Nordlund, Å. 1997, J. Geophys. Res., 102, 219

Hyman, J. M. 1979, Adv. Comp. Meth. for PDEs-III, ed. R. Vichnevetsky, \& R. S. Stepleman, Publ. IMACS, 313

Moffatt, H. 1978, Magnetic Field Generation in Electrically Conducting Fluids (Cambridge University Press)

Moffatt, H., \& Proctor, M. R. E. 1985, JFM, 154, 493

Nordlund, A., Brandenburg, A., Jennings, R. L., et al. 1992, ApJ, 392, 647

Parker, E. N. 1979, Cosmical Magnetic Fields (Oxford: Clarendon)

Podvigina, O., \& Pouquet, A. 1994, Phys. D, 75, 475

Tanner, S., \& Hughes, D. 1988, in Studia Geophysica et Geodaesia (Prague University Press), 343

Vainshtein, S. I., \& Cattaneo, F. 1992, ApJ, 393, 165

Zienicke, E., Politano, H., \& Pouquet, A. 1998, Phys. Rev. Lett., 81, 21 